\title{
Severe neonatal chlamydial conjunctivitis with unilateral corneal perforation after unattended home delivery in rural Ethiopia
}

\author{
Lorenzo Cirri, ${ }^{1}$ Abel Hailu, ${ }^{2}$ Frank Riedel ${ }^{2}$ \\ ${ }^{1}$ Arsi University, Asella; ${ }^{2}$ Department of Paediatrics and Child Health, Asella Teaching Hospital, Arsi University, Asella, \\ Ethiopia
}

\section{Summary}

Background. Neonatal chlamydial conjunctivitis is an easily preventable, though still highly prevalent infectious disease in low-income countries and if left untreated it is associated with major local and systemic complications that threat new-borns' health.

Case presentation. We present two cases of severe neonatal chlamydial conjunctivitis with unilateral corneal involvement in new-born children, after unattended home deliveries in rural Ethiopia. Despite antibiotic treatment, in one case, surgical corneal repair was necessary in order to avoid excessive scarring and subsequent unilateral blindness of the child.

Conclusions. As the presence of an ophthalmologist in sub-Saharan Africa is very rare, many children in the same condition may become blind due to lack of trained surgeons. For these reasons, screening programmes for sexually transmitted infections in pregnant women with suspect

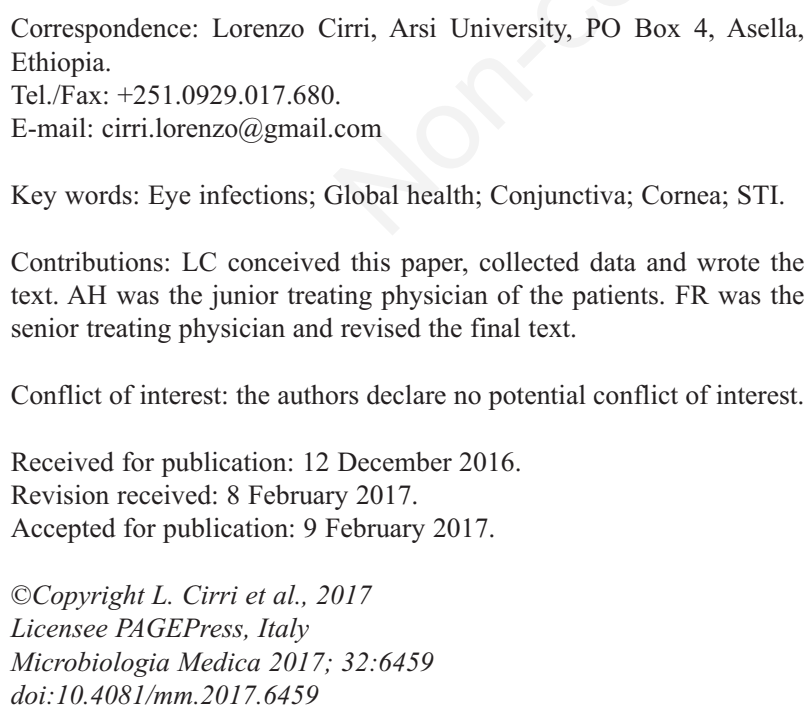

Conflict of interest: the authors declare no potential conflict of interest.

Received for publication: 12 December 2016.

Revision received: 8 February 2017.

Accepted for publication: 9 February 2017.

(C) Copyright L. Cirri et al., 2017

Licensee PAGEPress, Italy

Microbiologia Medica 2017; 32:6459

doi:10.4081/mm.2017.6459

This article is distributed under the terms of the Creative Commons Attribution Noncommercial License (by-nc 4.0) which permits any noncommercial use, distribution, and reproduction in any medium, provided the original author(s) and source are credited. vaginal discharge should be implemented by local authorities; as well as local prophylaxis with tetracyclin eye-ointment being made available in rural communities.

\section{Introduction}

Neonatal chlamydial conjunctivitis (NCC) is an easily preventable, though still highly prevalent infectious disease in developing countries. It is the most common eye disease in the first months after birth and often leads to systemic complications such as chlamydial pneumonia (5); if left untreated it is associated with a high morbidity and mortality (2). Chlamydial conjunctivitis is easy to distinguish from gonococcal conjunctivitis, as the former appears in the very first days after birth and the latter in the in the second week of life. Also, in contrast to gonococcal conjunctivitis, it is not often associated with local complications like corneal damage.

Prenatal screening and treatment of pregnant women is the best method for preventing chlamydial infection among neonates (9). Furthermore, there is some evidence $(6,10)$ that the application of tetracyclin eye-ointment (TEO) or eye-drops within one hour of birth may be effective in preventing NCC, as discussed later.

Still, as more than $80 \%$ of deliveries in our setting are home-based (3), most of which are unattended with no antenatal care (ANC) sought, neither STI-screenings nor prophylaxis with TEO are performed and the incidence of NCC remains high.

\section{Case Report}

Within a week, two new-born children aged 12-days-old and 14-days-old (Figure 1) were presented by their mothers to the paediatric emergency department of Asella Teaching Hospital (ATH), the main referral hospital of Arsi Zone in central Ethiopia, with a 24-hour history of unilateral mucopurulent eye-discharge, involving only the right eye of the 12-day-old child and the left eye in the 14-day-old child. The babies were both delivered at home in rural areas of the Arsi Zone, Oromia regional State, central Ethiopia. 

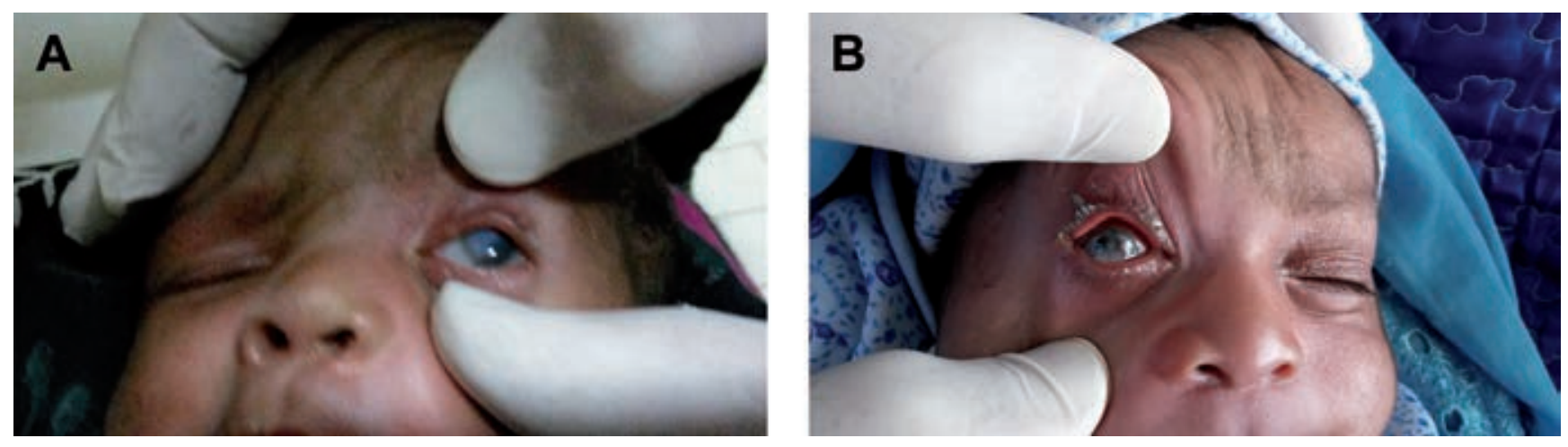

Figure 1. A) A 12-day-old-child after 1 day of antibiotic treatment; B) 14-day-old-child at presentation.

Neither mothers had received any form of antenatal care nor any help from traditional birth attendants during delivery; no screening tests for STIs, nor local tetracycline prophylaxis had therefore been performed. According to the mothers, the children had been in good health until approximately 3 days before, when they noticed rapidly progressing redness and oedema of the involved eye.

Upon presentation at the hospital, both children showed no sign or symptom of systemic disease; respiratory rate and temperature were normal and the feeding was regular. On clinical examination, the involved eyes of both children appeared swollen and the eyelids were kept closed by a partially crusted yellowish secretion. In contrast, the contralateral eye was open and looked completely normal. Upon manual opening of the eyelids and removal of the mucopurulent discharge, the conjunctiva appeared highly hyperaemic and the cornea appeared thinned, discoloured and damaged. Due to the late presentation of symptoms (over seven days after birth) a complicated congenital conjunctivitis, due to Chlamydia trachomatis, appeared more probable than congenital gonococcal conjunctivitis.

In order to confirm the suspected diagnosis an accurate history of the mothers was taken. As both mothers admitted having been suffering from vaginal discharge for many months, a trained nurse performed a gynaecological examination, including a vaginal swab. The test was performed in the Department of Clinical Laboratory of the Hirsch Institute of Tropical Medicine in Asella, Ethiopia, using a rapid immunochromatographic test, with a positive result for chlamydial antigen in both children. Results from a gonorrhoea rapid test were negative. Together with the typical medical history, both children were consequently diagnosed with severe neonatal chlamydial conjunctivitis with corneal involvement and were promptly started on systemic antibiotic treatment with Erythromycin $50 \mathrm{mg} / \mathrm{kg}$ /day orally divided into 4 doses daily for 14 days (1). Local tetracyclin ointment had already been started on admission, therefore no test was performed directly on conjunctival discharge. After one week of antibiotics and reduced swelling, in one case a surgical corneal repair was necessary in order to remove necrot- ic tissue, avoid excessive scarring, and therefore unilateral blindness of the child due to corneal perforation.

\section{Discussion and Conclusions}

The described cases show a very common scenario in a paediatric ward in low-income countries, where NCC is very prevalent, although precise data about its epidemiology in sub-Saharan Africa, especially in rural Ethiopia are not available. Nevertheless, the cases are interesting because of the severity of the local complications, as well as the unilateral location of the infection.

NCC is not usually associated with corneal damage or perforation, in contrast to neonatal conjunctivitis due to Neisseria gonorrhoea (8) or Herpes simplex (4). A late presentation to the hospital, together with a lack of antenatal care and an unattended home delivery, without any local prophylaxis, may all be associated with the severity of the presentation. Moreover, our patients presented a unilateral eye involvement with a totally normal contralateral eye, whereas ophtalmia neonatorum is typically associated with bilateral eye involvement due to the close anatomical connection with the infected birth canal during delivery.

Due to appropriate treatment and special surgical repair, our patients may be able to see again with the involved eye. However the presence of an ophthalmologist is very rare in sub-saharan Africa and many children in the same condition may become blind due to the lack of trained surgeons. From a public health prospective, local authorities should therefore invest on prevention of NCC rather than its complicated surgical treatment, especially through the implementation of outreach screening programmes for STIs in pregnancy.

The use of TEO in the prophylaxis of NCC is argued. Although a meta-analysis by Darling at al. (1) showed that neither tetracycline- nor erythromycin-based prophylactic regimens are effective in the prevention of NCC, national Ethiopian guidelines (7) still recommend the use TEO based on previous evidence $(6,10)$. 
More studies about the exact epidemiology, long term complications and prophylaxis of NCC in low-income settings are needed.

\section{References}

1. Darling EK, McDonald H. A meta-analysis of the efficacy of ocular prophylactic agents used for the prevention of gonococcal and Chlamydial Ophthalmia Neonatorum. J Midwifery Womens Health 2010;55:319-27.

2. Datta P, Laga M, Plummer FA, et al. Infection and disease after perinatal exposure to Chlamydia trachomatis in Nairobi, Kenya. J Infect Dis 1988;158:524-8.

3. Fikre AA, Demissie M. Prevalence of institutional delivery and associated factors in Dodota Woreda (district), Oromia regional state, Ethiopia. Reprod Health 2012;9:33.

4. Gao H, Jia Y, Li S, et al. Conjunctival flap covering combined with antiviral and ster-oid therapy for severe herpes simplex virus necrotizing stromal keratitis. Sci World J 2015;2015:565964.

5. Kakar S, Bhalla P, Maria A, et al. Chlamydia trachomatis causing neonatal conjunctivi-tis in a tertiary care center. Indian J Med Microbiol 2010;28:45-7.

6. Laga M, Plummer F, Piot P, et al. Prophylaxis of gonococcal and chlamydial Ophthal-mia neonatorum. N Engl J Med 1988:318:653-7.

7. Ministry of Health of Ethiopia. Neonatal intensive care unit management protocol. 2014. Available from: http://www.epseth.org/a/files/Status\%20of\%20NICU\% 20in\%20Ethiopia.pdf

8. Tipple C, Smith A, Bakowska E, et al. Corneal perforation requiring corneal grafting: a rare complication of gonococcal eye infection. Sex Transm Infect 2010;86:447-8.

9.Zar HJ. Neonatal chlamydial infection: prevention and treatment. Pediatr Drugs 2005;7:103.

10. Workowski KA, Bolan GA. Centers for Disease Control and Prevention (CDC). Sex-ually transmitted diseases treatment guidelines. MMWR Recomm Rep 2015;64:1-138. 